\title{
DIFERENTES CONCENTRAÇÕES DE ZINCO NO DESENVOLVI- MENTO DE PLANTAS DE PHASEOLUS VULGARIS L.
}

\author{
Menegatti RD*, Pereira AS $S^{* *}$, Oliveira $L^{* * *}$, Dorneles $\operatorname{AOS}^{* * *}$, Dutra ${ }^{* * * * *}$, Deuner $S^{* * * * * *}$
}

\begin{abstract}
Resumo
Objetivou-se com este estudo avaliar os efeitos da aplicação de solução nutritiva com concentrações crescentes de zinco (Zn) no desenvolvimento inicial e no teor de pigmentos de plantas de feijão cultivadas em condições de casa de vegetação. O experimento foi desenvolvido no Laboratório de Nutrição de Plantas (LNP) e em casa de vegetação pertencentes ao Departamento de Botânica da Universidade Federal de Pelotas (UFPel). A semeadura foi realizada em vasos preenchidos com areia média lavada. Sete dias após a emergência das plântulas foi aplicada, com intervalos de dois dias, solução nutritiva

\footnotetext{
* Mestre em Produção Vegetal pela Universidade do Estado de Santa Catarina; Pós-graduada em Docência no Ensino Superior pela Universidade do Contestado; doutoranda do Programa de Pós-graduação em Fisiologia Vegetal pela Universidade Federal de Pelotas; renata.d.menegatti@gmail.com

** Mestre em Agrobiologia pela Universidade Federal de Santa Maria; Graduada em Ciências Biológicas pela Universidade de Santa Maria; lyne_asp@hotmail.com

** Graduada em Ciências Biológicas pela Universidade da Região da Campanha; mestranda do Programa de Pós-graduação em Fisiologia Vegetal pela Universidade Federal de Pelotas; luanadasoliveiras@hotmail.com

${ }^{* * * *}$ Mestre em Agrobiologia pela Universidade Federal de Santa Maria; doutorando do Programa de Pós-graduação em Fisiologia Vegetal pela Universidade Federal de Pelotas; athos_odin@hotmail.com

${ }^{* * * * *}$ Graduada em Ciências Biológicas pela Universidade Federal de Pelotas; mestranda do Programa de Pós-graduação em Fisiologia Vegetal pela Universidade Federal de Pelotas; dbarwaldtdutra@hotmail.com

${ }_{* * * * * *}$ Doutor em Agronomia pela Universidade Federal de Lavras; Mestre em Fisiologia Vegetal pela Universidade Federal de Pelotas; Professor do Programa de Pós-graduação em Fisiologia Vegetal da Universidade Federal de Pelotas; sdeuner@yahoo.com.br
} 
com quatro concentrações crescentes de $\mathrm{Zn}(2,50,75$ e $100 \mu \mathrm{M})$, dispostas em um delineamento experimental inteiramente casualizado com quatro repetições, sendo cada uma delas composta por seis plantas. Aos 45 dias após a emergência das plântulas, avaliaram-se as seguintes variáveis: altura das plantas, diâmetro do caule, número de folhas, área foliar e comprimento de raiz, além do índice de pigmentos fotossintéticos. Com o aumento das concentrações de $\mathrm{Zn}$ verificou-se decréscimo significativo para as variáveis diâmetro do caule e comprimento de raiz, sugerindo que maiores concentrações de Zn podem influenciar negativamente no desenvolvimento das plantas de feijão. Os índices de clorofila e flavonoides apresentaram elevação com o aumento das doses de Zn. Esse efeito possivelmente está relacionado a uma reação estratégica da espécie, que visa minimizar o efeito tóxico das concentrações crescentes de Zn no desenvolvimento das plantas.

Palavras-chave: Feijão. Micronutriente. Pigmentos foliares. Toxicidade.

\title{
Different zinc concentrations on the development of Phaseolus vulgaris L. plants
}

\begin{abstract}
The objective of this study was to evaluate the effects of nutritive solution with increasing concentrations of zinc $(\mathrm{Zn})$ on development and pigment content of bean plants grown under greenhouse conditions. The experiment was carried out in the Laboratory of Plant Nutrition (LNP) and in a greenhouse belonging to the Department of Botany of the Federal University of Pelotas (UFPel). The bean sowing was carried out in pots filled with washed medium sand. Seven days after seedling emergence the nutritive solution was applied with four different concentrations of $\mathrm{Zn}(2,50,75$ and $100 \mu \mathrm{M})$ arranged in a completely randomized experimental design with four replicates of six plants per treatment. At 45 days after emergence, the experiment was finalized and the following variables were evaluated: shoot height, stalk diameter, leaf number, leaf area and root length, as well as determination of photosynthetic pigments. With increase of Zn concentrations, there was a significant decrease for variables: stem diameter and root length, suggesting that higher concentrations of Zn may negatively influence the development of bean plants. Chlorophyll and flavonoid indexes were elevated with increasing doses of $\mathrm{Zn}$. This effect is possibly related to strategic reaction of plant species, which aims to minimize the toxic effects of increasing concentrations of Zn on plant development.
\end{abstract}

Keywords: Bean. Micronutrient. Leaf pigments. Toxicity.

\section{INTRODUÇÃO}

O zinco $(\mathrm{Zn})$ é um micronutriente de grande influência na produtividade da cultura do feijão (Phaseolus vulgaris L.). De acordo com Santos, ${ }^{1}$ os principais sintomas de deficiência desse micronutriente são a clorose, presente em folhas mais novas, e a redução do crescimento radicular, que torna a planta incapaz de absorver quantidades mínimas de Zn, necessárias para o seu desenvolvimento e 
que influenciará diretamente na produtividade. Já o excesso desse elemento afeta negativamente os processos fisiológicos, limitando o crescimento e o desenvolvimento das plantas, além da redução da produção de biomassa e grãos. ${ }^{2}$

O excesso de Zn em áreas agricultáveis ocorre principalmente por meio da aplicação de produtos que contenham este elemento químico em sua formulação, como, por exemplo, herbicidas, fungicidas e fertilizantes fosfatados, bem como a partir do despejo direto de dejetos suínos e lodo de esgoto. ${ }^{3,4}$ A presença dos compostos mencionados anteriormente nos solos, ao longo do tempo, poderá acarretar problemas de fitotoxidade em plantas e, mais tarde, poderá tornar a atividade agrícola quase impraticável. ${ }^{5}$

De modo geral, cada nutriente tem funções específicas nas plantas, e seus excessos e limitações podem produzir sintomas de deficiência ou de toxidez. ${ }^{6}$ A presença de Zn em áreas agrícolas atualmente é um tema referenciado junto aos grandes problemas ambientais, em razão de que, por ser um metal pesado, ao atingir o solo, pode desencadear uma série de modificações no ecossistema (fauna, flora, águas superficiais e subterrâneas). ${ }^{5,7}$

Para a cultura do feijão não foram realizadas pesquisas pontuais que determinem qual a dose tóxica de Zn nos solos, ou seja, qual o limite tolerável da presença do elemento que não prejudicará o desenvolvimento da planta e não induzirá sintomas e processos de estresse. Porém, estudos ressaltam que o aparecimento de pigmentos pardo-avermelhados e clorose nas folhas é um forte indicador de toxidez por Zn, e que, possivelmente, esses sintomas podem estar relacionados com atividades do metabolismo secundário. ${ }^{8,9}$

Concentrações supra-ótimas de Zn podem alterar não somente o desenvolvimento das plantas e a produtividade, mas também modificar os processos bioquímicos e metabólicos, alterar as taxas fotossintéticas, e outros. ${ }^{10}$ Segundo Santos, ${ }^{1}$ altos níveis de Zn são prejudiciais à cultura do café, pois reduzem a taxa de crescimento das plantas, assim como a massa foliar. O mesmo autor ainda relatou a possível alteração na atividade fotossintética pela redução dos níveis de clorofila em razão da toxidez por Zn.

A massa vegetal, assim como a área foliar de algumas culturas agrícolas, entre elas o milho e o feijão, são consideradas fontes determinantes para a geração de fotoassimilados para a planta e, portanto, alterações e reduções dessas fontes poderão refletir na baixa do crescimento da planta e na menor produção de grãos. ${ }^{11,12,8}$

Já a presença e abundância de pigmentos fotossintéticos refletem diretamente na qualidade da planta, considerando que plantas cultivadas em altas concentrações de Zn apresentam dificuldade na distribuição de seiva bruta, o que acarreta a redução da concentração de clorofila $a$ e $b$ e de proteínas solúveis, ${ }^{13}$ desencadeando efeitos depressivos nas variáveis de crescimento da planta e deficiências no processo fotossintético. ${ }^{8}$

A determinação do nível supra-ótimo de Zn para a cultura do feijão poderá gerar alternativas para a ocupação de áreas com maiores concentrações do nutriente, de modo a propor a revegetação de áreas consideradas impraticáveis ao uso agrícola. ${ }^{14,15,5,16}$ Atualmente são poucos os estudos que empregam a cultura do feijão em solos com excesso de $\mathrm{Zn}$ e pouco se conhece especificamente sobre o desenvolvimento de plantas nessas condições. 
Assim, o objetivo com este trabalho foi avaliar os efeitos da aplicação de concentrações crescentes de Zn no desenvolvimento de plantas de feijão cultivadas em casa de vegetação.

\section{MATERIAL E MÉTODOS}

O experimento foi desenvolvido no Laboratório de Nutrição de Plantas (LNP) e em casa de vegetação pertencente ao Departamento de Botânica no Instituto de Biologia na Universidade Federal de Pelotas, localizado no Município de Capão do Leão, RS. A temperatura média da casa de vegetação durante a realização do estudo foi de $25^{\circ} \mathrm{C}$, e a umidade relativa era de $80 \%$.

O delineamento experimental utilizado foi o inteiramente casualizado, com quatro diferentes concentrações de Zn em solução nutritiva de Hoagland, sendo o tratamento controle ( $2 \mu \mathrm{M}$ de $\mathrm{Zn}$ ) e os demais tratamentos $(25,50$ e $75 \mu \mathrm{M}$ de $\mathrm{Zn})$ com quatro repetições cada; cada repetição composta por seis plantas. A solução nutritiva de Hoagland tem em sua composição (em $\mu \mathrm{M}$ ): 6090,5 de N; 974,3 de Mg; 4986,7 de Cl; 2679,2 de K; 2436,2 de Ca; 359,9 de S; 243,6 de P; 0,5 de Cu; 2,0 de Mn; 2,0 de Zn; 0,17 de Ni; 24,9 de B; 0,5 de Mo; 47,9 de Fe (FeSO4/Na-EDTA) e pH de 5,8. ${ }^{17}$

Primeiramente, sementes de feijão foram semeadas em recipientes plásticos contendo areia lavada e acondicionados em casa de vegetação. Sete dias após a semeadura, as plantas foram expostas aos diferentes tratamentos, recebendo uma nova rega a cada dois dias até o final do experimento, que ocorreu 45 dias após a implantação.

Aos 45 dias após a semeadura analisaram-se as seguintes variáveis: altura das plantas (mm) e diâmetro do caule ( $\mathrm{mm}$ ), utilizando-se o paquímetro digital; comprimento de raiz (mm), com uso de uma régua milimetrada; número de folhas por planta. A área foliar foi obtida a partir do processo de digitalização das folhas com o auxílio de um scanner modelo Canon Pixma MG2410, e as imagens adquiridas foram processadas por meio do software Image $^{\oplus}$.

Para a análise estatística dos dados, primeiramente foi verificada a normalidade da distribuição dos erros mediante o teste de Anderson-Darling e a homogeneidade das variâncias dos erros por meio do teste de Bartlett $(\mathrm{p}<0,05)$. As médias de todas as variáveis foram submetidas à análise de variância e comparadas pelo teste de Scott-Knott; por fim, as variáveis que apresentaram diferenças significativas foram submetidas à análise de regressão, ambas ao nível de $5 \%$ de probabilidade de erro. A análise dos dados foi realizada no pacote estatístico SISVAR 5.3. ${ }^{18}$

\section{RESULTADOS E DISCUSSÃO}

De acordo com a análise de variância, foi possível verificar efeitos significativos das concentrações crescentes de Zn, exceto para as variáveis de altura de plantas e área foliar (Tabela 1). 
Tabela 1 - Altura, diâmetro do caule, número de folhas, área foliar e comprimento de raiz de plantas de feijão cultivadas em solução nutritiva com concentrações crescentes de $\mathrm{Zn}(2,25,50 \text { e } 75 \mu \mathrm{M})^{\dagger}$

\begin{tabular}{|c|c|c|c|c|c|}
\hline $\begin{array}{l}\text { Tratamento } \\
\qquad(\mu \mathrm{M})\end{array}$ & $\begin{array}{l}\text { Altura } \\
(\mathrm{cm})\end{array}$ & $\begin{array}{c}\text { Diâmetro do } \\
\text { caule } \\
(\mathbf{m m})\end{array}$ & No folhas & $\begin{array}{c}\text { Área foliar } \\
\left(\mathrm{cm}^{2}\right)\end{array}$ & $\begin{array}{l}\text { Comp. raiz } \\
\quad(\mathrm{cm})\end{array}$ \\
\hline 2 & $13.2 \pm 0.5 \mathrm{~A}$ & $2.7 \pm 0.06 \mathrm{~A}$ & $5.0 \pm 0.1 \mathrm{~B}$ & $69 \pm 2.3 \mathrm{~A}$ & $18.2 \pm 0.9 \mathrm{~A}$ \\
\hline 25 & $12.5 \pm 0.6 \mathrm{~A}$ & $2.5 \pm 0.17 \mathrm{~A}$ & $5.0 \pm 0.2 \mathrm{~B}$ & $67 \pm 2.5 \mathrm{~A}$ & $18.1 \pm 0.6 \mathrm{~A}$ \\
\hline 50 & $13.9 \pm 0.7 \mathrm{~A}$ & $2.1 \pm 0.24 \mathrm{~B}$ & $5.0 \pm 0.2 \mathrm{~B}$ & $72 \pm 1.8 \mathrm{~A}$ & $14.6 \pm 0.3 \mathrm{~B}$ \\
\hline 75 & $14.3 \pm 0.1 \mathrm{~A}$ & $2.1 \pm 0.12 \mathrm{~B}$ & $5.7 \pm 0.3 \mathrm{~A}$ & $70 \pm 3.3 \mathrm{~A}$ & $14.8 \pm 0.5 \mathrm{~B}$ \\
\hline
\end{tabular}

Plantas submetidas a $25 \mu \mathrm{M}$ de Zn não diferiram do tratamento controle para diâmetro do caule, porém os demais tratamentos apresentaram significativa redução nessa variável resposta (Figura 1). Esses resultados sugerem que concentrações de $\mathrm{Zn}$ superiores a $25 \mu \mathrm{M}$ tem efeito negativo sobre a variável diâmetro de caule.

Acredita-se que plantas com maior diâmetro de caule proporcionam melhor desempenho a campo e maiores condições de sobrevivência. ${ }^{19}$ De acordo com pesquisa realizada, ${ }^{20}$ quanto maior o diâmetro do caule maior a alocação de fotoassimilados na parte aérea, e, como consequência, a capacidade de sobrevivência da planta será superior em condições de campo.

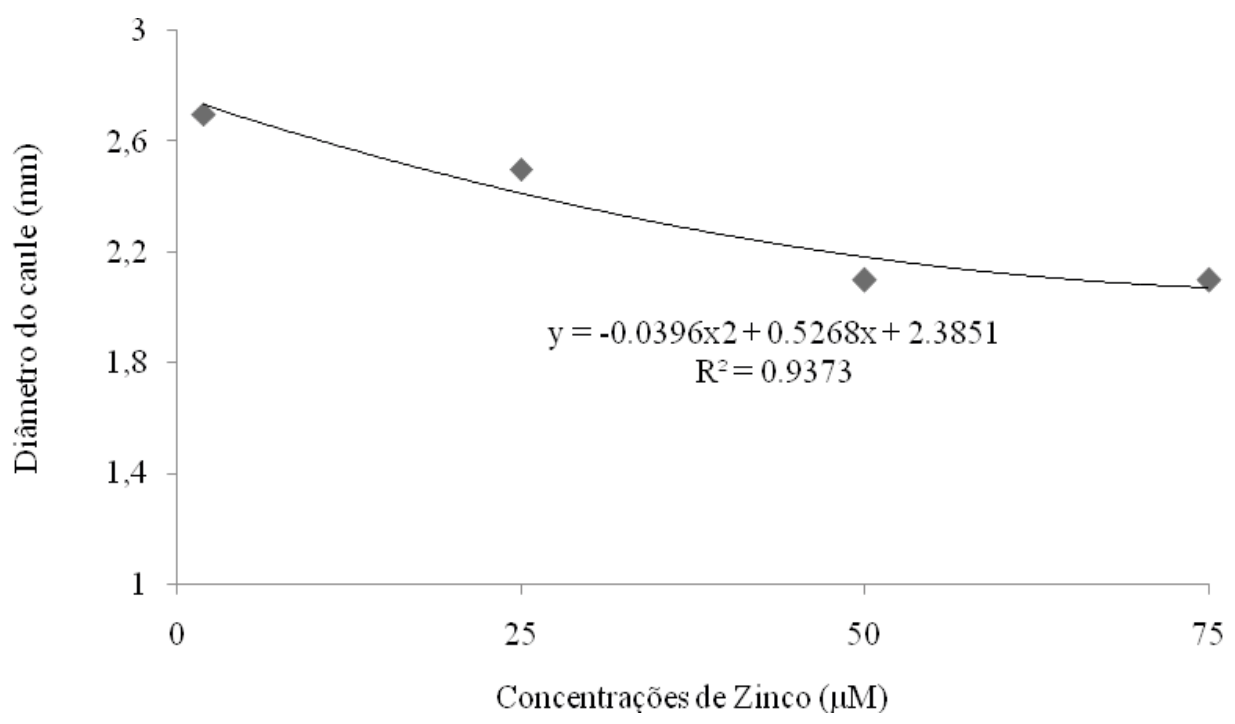

Figura 1 - Diâmetro do caule de plantas de feijão submetidas a concentrações crescentes de Zn na solução nutritiva

Plantas expostas a concentrações menores de $\mathrm{Zn}(2,25,50 \mu \mathrm{M})$ apresentaram menor número de folhas por planta quando comparadas às plantas expostas a concentração mais elevada (75 $\mu \mathrm{M})$ (Figura 2). Esse aumento no número de folhas no tratamento com maior concentração de Zn pode ser em decorrência de a planta estar sob estresse, gerando uma nova folha de forma a aumentar a área foliar e a capacidade fotossintética, tentando otimizar o provimento de energia.

\footnotetext{
${ }^{\dagger}$ Letras diferentes indicam diferença significativa entre os tratamentos pelo teste de Scott-Knott ( $\left.\mathrm{p} \leq 5 \%\right)$.
} 


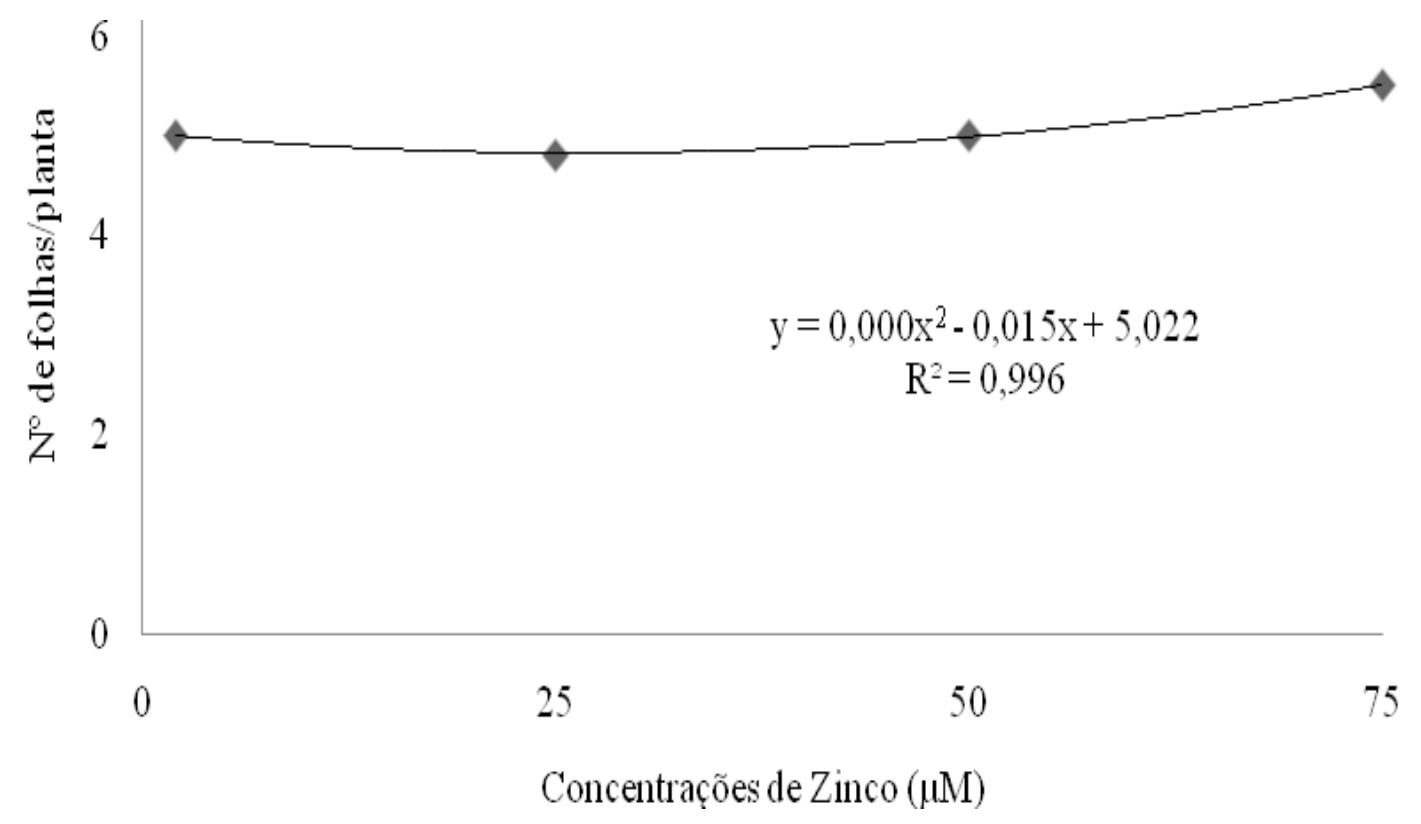

Figura 2 - Número de folhas por plantas de feijão submetidas a concentrações crescentes de Zn na solução nutritiva

Na Figura 3 fica evidente o decréscimo de raízes em relação ao aumento da disponibilidade de $\mathrm{Zn}$ na solução, indicando que maiores concentrações do elemento podem influenciar negativamente o desenvolvimento das raízes de feijão. Além disso, a produção de raízes menores pode diminuir a eficiência de aquisição, translocação e utilização de nutrientes do solo, prejudicando o desenvolvimento das plantas.

Quando a concentração de Zn disponível para a planta excede níveis ótimos, nota-se diminuição do crescimento, ${ }^{21,22}$ inibição do desenvolvimento radicular e clorose nas folhas em plantas de Brassica spp. ${ }^{23}$ Observações iguais a essa foram encontradas no presente estudo, evidenciando-se a suscetibilidade do feijoeiro a concentrações crescentes de Zn.

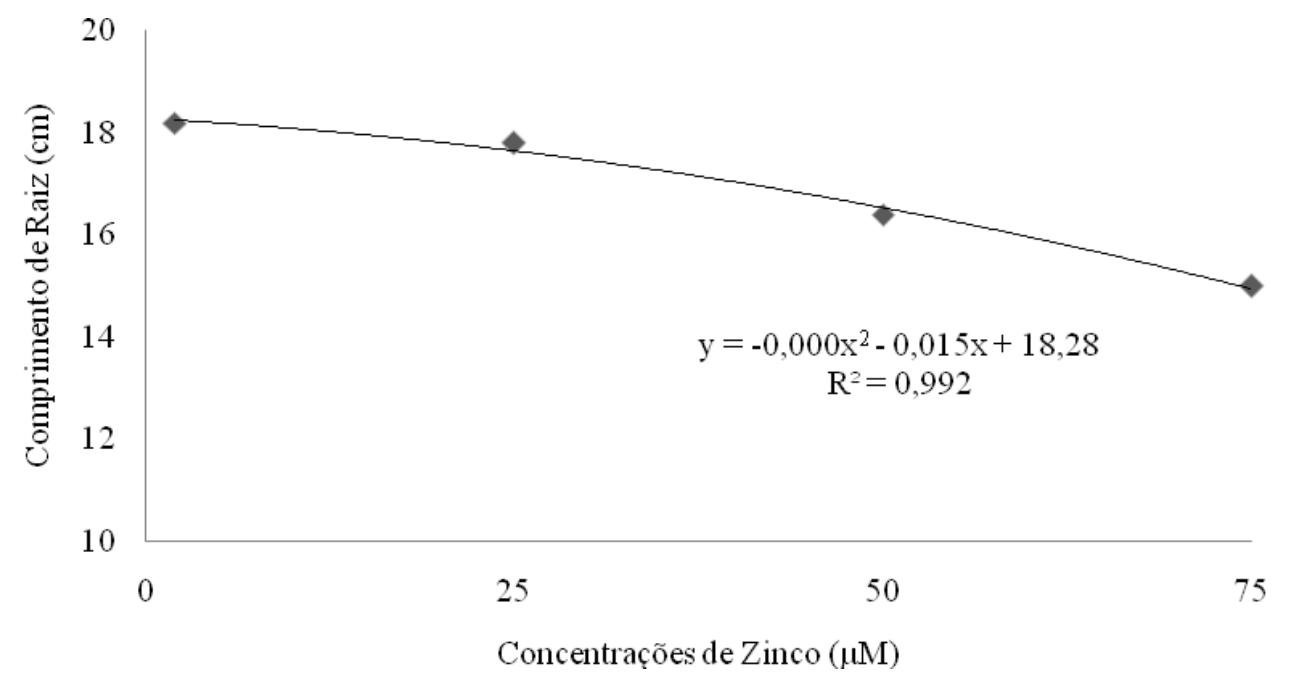

Figura 3 - Comprimento de raiz de plantas de feijão submetidas a concentrações crescentes de Zn na solução nutritiva 
Estudos recentes sugerem que o efeito de toxicidade do Zn depende da espécie vegetal e do estádio de crescimento da planta, e que a inibição do crescimento e a redução da biomassa são respostas comuns das plantas ao excesso de $\mathrm{Zn} .{ }^{24,25,26}$ Acredita-se, a partir dos resultados deste trabalho, que a redução no diâmetro do caule e o comprimento de raiz nas plantas de feijão são resultados do estresse oxidativo induzido pelo excesso de $\mathrm{Zn} .^{27,28}$

O estresse por altas doses de Zinco também é responsável por alterações no aparato fotossintético. ${ }^{29}$ Os índices de clorofila e flavonoides (Tabela 2) apresentaram elevação com o aumento das doses de Zn até a concentração de $50 \mu \mathrm{M}$ e, possivelmente, as plantas apresentaram essas respostas na tentativa de mitigar os efeitos tóxicos do Zn em nível celular.

No entanto, essas respostas não foram suficientes, em razão do decréscimo no crescimento das plantas frente às concentrações crescentes de $\mathrm{Zn}$. Resultados similares foram encontrados em estudo realizado com mamona (Ricinus communis $c v$. BRS Energia), ${ }^{24}$ no qual o índice de clorofila apresentou elevação com as doses de Zn testadas, e em experimento conduzido com feijoeiro, no qual os teores de clorofila não foram influenciados pela adição de $\mathrm{Zn} .^{30}$

Tabela 2 - Teores de clorofila, flavonoides, antocianinas e nitrogênio em folhas de feijão submetidas a concentrações crescentes de Zn na solução nutritiva ${ }^{\ddagger}$

\begin{tabular}{cccc}
\hline $\begin{array}{c}\text { Tratamento } \\
(\mu \mathrm{M})\end{array}$ & Teor de clorofila & Teor de flavonoides & Teor de antocianina \\
\hline 2 & $17.68 \pm 0.69 \mathrm{~B}$ & $0.86 \pm 0.07 \mathrm{C}$ & $0.18 \pm 0.005 \mathrm{~A}$ \\
25 & $17.63 \pm 0.63 \mathrm{~B}$ & $1.11 \pm 0.03 \mathrm{~B}$ & $0.18 \pm 0.001 \mathrm{~A}$ \\
50 & $21.77 \pm 0.70 \mathrm{~A}$ & $1.74 \pm 0.03 \mathrm{~A}$ & $0.17 \pm 0.007 \mathrm{~A}$ \\
75 & $18.18 \pm 0.71 \mathrm{~B}$ & $0.90 \pm 0.02 \mathrm{C}$ & $0.17 \pm 0.002 \mathrm{~A}$ \\
\hline
\end{tabular}

O teor de antocianina não sofreu alterações significativas (Tabela 2). As antocianinas podem atuar como quelante de metais, em espécies de Grevillea spp. tolerantes à salinidade, o teor desses pigmentos manteve-se alto quando as plantas foram expostas a condições de estresse. ${ }^{31}$

\section{CONCLUSÃO}

O presente estudo evidencia que maiores concentrações de Zn podem influenciar negativamente o desenvolvimento das plantas de feijão.

Concentrações de $\mathrm{Zn}$ acima de dois $\mu \mathrm{M}$ podem ser prejudiciais ao feijoeiro. As respostas do aparato fotossintético, ou seja, o aumento dos índices de clorofila e flavonoides com a elevação das doses de Zn sugere ser uma estratégia da espécie para minimizar o efeito das concentrações crescentes de Zn no desenvolvimento das plantas.

${ }^{\ddagger}$ Letras diferentes indicam diferença significativa entre os tratamentos pelo teste de Scott-Knott ( $\left.\mathrm{p} \leq 5 \%\right)$. 


\section{REFERÊNCIAS}

1. Santos JO. Deficiência e excesso de zinco em mudas de cafeeiro: metabolismo de carboidratos e respostas antioxidantes [dissertação]. Minas Gerais: Universidade Federal de Lavras; 2014.

2. Sagardoy R, Vázquez S, Florez-Sarasa ID, Albacete A, Ribas-Carbó M, Flexas J, et al. Stomatalandmesophyllconductancesto $\mathrm{CO}_{2}$ are themainlimitationstophotosynthesisin sugar beet (Beta vulgaris) plantsgrownwithexcesszinc. New Phytologist 2010;187(1):145-58.

3. Fageria KN. Níveis adequados e tóxicos de zinco na produção de arroz, feijão, milho, soja e trigo em solo de cerrado. Rev. Bras. Eng. Agríc. Ambient. 2000;4(3):390-5.

4. Borges JR, Mello JWV, Schaefer CEGR, Dussin TM, Abrahão WAP. Valores de referência local e avaliação da contaminação por zinco em solos adjacentes a áreas mineradas no município de Vazante-MG. Rev. Bras. Ciênc. Solo 2008;32:2883-93.

5. Alexandre JR, Oliveira MLF, Santos TC, Canton GC, Conceição JM, Eutropio FJ, et al. Zinco e ferro: de micronutrientes a contaminantes do solo. Natureza online 2012;10(1):23-8.

6. Hooda PS. Trace elements in soils. United Kingdom: Wiley-Blackwell; 2010.

7. Vale MS, Abreu KV, Gouveia ST, Leitão RC, Santaella ST. Efeito da toxicidade de Cr (VI) e Zn (II) no crescimento do fungo filamentoso Aspergillusniger isolado de efluente industrial. Eng. Sanit. Ambient. 2011;16(1):237-44.

8. Silva TMR, Prado MR, Vale WD, Avalhães CC, Puga A, Fonseca MI. Toxicidade do zinco em milheto cultivado em Latossolo Vermelho Distrófico. Rev. Bras. Ciênc. Agrár. 2010;5(3):336-40.

9. Lincoln T, Zeigel E. Fisiologia Vegetal. 4a ed. Porto Alegre: Artmed, 2013.

10. Oliveira IP, Oliveira LC. Metais pesados. Revista Eletrônica Faculdade de Iporá 2011;1:59-86.

11. Magalhães PC, Jones R. Aumento de fotoassimilados na taxa de crescimento e peso final dos grãos de milho. Pesq. Agropec. Bras. 1990;25(12):1747-54.

12. Magalhães PC, Silva JB, Duraes FOM. Fitotoxidade de herbicidas aplicados em pós-emergência na fase inicial da cultura do milho. Planta daninha 2000;18(2):277-84.

13. Khurana N, Chatterjee C. Influence of variable zinc on yield, oil content, and physiology of sunflower. Comm Soil Sci Plant Anal. 2001;32(19):323-30.

14. García G, Faz A, Cunha M. Performance of Piptatherum miliaceum (Smilo grass) in edaphic Pb and $\mathrm{Zn}$ phytoremediation over a short growth period. Int. Biodeterior. Biodegrad. 2004;54:245-50.

15. Vaillant N, Monnet F, Hitmi A, Sallanon H, Coudret A. Comparative study of responses in four Datura species to a zinc stress. Chemosphere 2005;59(7):1005-13. 
16. Silva FR da, Antoniolli IZ, Weirich WS, Dellai A, Missio LE, Scheid D. Potencial da associação pisolithusmicrocarpus com mudas de corymbiacitriodora em solo contaminado com zinco. Ci. Fl. 2016;26(1):181-91.

17. Hoagland DR, Arnon DI. The water culture method for growing plants without soil. Berkeley, California: The college of agriculture, Univ. of California; 1950.

18. Ferreira DF. Sisvar: a computerstatisticalanalysis system. Ciênc. Agrotec. 2011;35(6):1039-42.

19. Câmara GMS, Godoy OP. Desempenho vegetativo produtivo de cultivares de mandioca (Manihot esculenta Crantz) a partir de manivas com diferentes diâmetros. Sci. Agric. 1998;55(2):25-42.

20. Scalon SPQ, Scalon HF, Rigoni MR, Verado F. Germinação e crescimento de mudas de pitangueira (Eugenia uniflora L.) sob condições de sombreamento. Rev. Bras. Frutic. 2001;23(3):652-5.

21. Zampieri MCT, Saiki M, Tavares AR, Pinna GFAM. Acúmulo de minerais em Aechmea blanchetiana (Baker) L.B. Smith (Bromeliaceae) contaminadas com zinco em cultivo in vitro. Hoehnea 2012;39(3):379-85.

22. Lin Y, Aarts MGM. The molecular mechanism of zinc and cadmium stress response in plants. Cell. Mol. Life Sci. 2012;69:3187-206.

23. Ebbs SD, Kochian LV. Toxicity of zinc and copper to Brassica species: implications for phytoremediation. J. Environ. Qual. Madison 1997;26(3):776-81.

24. Marques MC, Nascimento CWA. Tolerância de mamona a zinco avaliada por fluorescência de clorofila e nutrição das plantas. Rev. Bras. Ciênc. Solo 2014;38(3):850-7.

25. Alonso-Blázquez N, García-Gómez C, Fernández MD. Influence of Zn-contaminated soils in the antioxidativedefence system of wheat (Triticumaestivum) and maize (Zea mays) at different exposure times: potential use as biomarkers. Ecotoxicology 2015;24(2):279-91.

26. Bernardy K, Farias JG, Dorneles AOS, Pereira AS, Schorr MRW, Thewes FR, et al. Changes in root morphology and dry matter production in Pfaffiaglomerata (Spreng.) Pedersen accessions in response to excessive zinc. Rev. Bras. Plantas Med. 2016;18(2):613-20.

27. Rao KVM, Sresty TVS. Antioxidative parameters in the seedlings of pigeonpea (Cajanuscajan L. Millspaugh) in response to Zn and Ni stresses. Plant Science 2000;157(1):113-28.

28. Remans T, Opdenakker K, Guisez Y, Carleer R, Schat H, Vangronsveld J, Cuypers A. Exposure of Arabidopsis thaliana to excess $\mathrm{Zn}$ reveals a Zn specific oxidative stress signature. Environ. Exp. Bot. 2012;84(1):61-71.

29. Kurdziel B, Prasad MNV, Strzalka K. Photosynthesis in heavy metal stressed plants. In: Prasad MNV. Heavy metal stress in plants: from biomolecules to ecosystems. New York: Springer; 2002. p. 146-81. 
30. Teixeira IR, Borém A, Andrade MJB, Giúdice MPD, Cecon PR. Fontes e doses de zinco no feijoeiro cultivado em diferentes épocas de semeadura. Acta Sci., Agron. 2008;30(2):255-9.

31. Kennedy BF, De Fillippis LF. Physiological and oxidative response to $\mathrm{NaCl}$ of the salt tolerant Grevillea ilicifolia and the salt sensitive Grevillea arenaria. J. Plant Physiol. 1999;155:746-54.

Data da submissão: 29 de dezembro de 2016 Avaliado em: 01 de fevereiro de 2017 (AVALIADOR A) Avaliado em: 03 de fevereiro de 2017 (AVALIADOR B) Aceito em: 27 de abril de 2017 